\title{
Vacuum polarization near asymptotically anti-de Sitter black holes in odd dimensions
}

\author{
Kiyoshi Shiraishi \\ Akita Junior College, Shimokitade-Sakura, \\ Akita-shi, Akita 010, Japan \\ and \\ Takuya Maki \\ Department of Physics, Tokyo Metropolitan University, \\ Minami-ohsawa, Hachioji-shi, Tokyo 192-03, Japan
}

Class. Quantum Grav. 11 (1994) pp. 1687-1696

\begin{abstract}
Recently, Bañados, Teitelboim and Zanelli obtained spherically symmetric black hole solutions in a particular class of Einstein-Lovelock gravity. We derive the propagator in an exact form for a conformal scalar field in the asymptotically anti-de Sitter black hole spacetime so as to study the quantum effects of the scalar fields. We treat the cases in odd dimensions in this paper. We calculate the vacuum expectation value of $\left\langle\varphi^{2}\right\rangle$ and show its dependence on the radial coordinate for the five-dimensional case as an example.

PACS numbers: 0450, 0462, 9760L
\end{abstract}

\section{Introduction}

For a couple of decades quantum field theorists have studied the quantum field near black holes $[1,2,3]$. Study of the quantization in the black hole background is important not only because it is necessary to attain the complete description of black hole thermodynamics but also because the quantum back reaction may change the picture of the endpoint of black hole evaporation.

Recently, the black hole solution to odd-dimensional Einstein-Lovelock gravity has been found and the global structure of the spacetime and thermodynamics at 'zero-loop' level have been analysed ${ }^{1}[4]$. The black hole solution in odd dimensions approaches anti-de Sitter space in the asymptotic region and is the higher-dimensional generalization of the three-dimensional black hole solution $[5,6,7,8,9]$. It will be interesting to investigate the nature of the quantum

\footnotetext{
${ }^{1}$ Hereafter, we call the black hole solution found by them the 'Bañados' black hole', simply for reasons of brevity.
} 
field in the odd-dimensional black hole background, because in odd dimensions there is no conformal anomaly at the one-loop level [3], which is believed to be closely connected with Hawking radiation and is expected to have something to do with other quantum effects near the black holes at least in four dimensions.

The present authors [7] and several groups [8,9] have obtained the propagator and the vacuum expectation value of $\left\langle\varphi^{2}\right\rangle$ and stress tensor for a conformally coupled scalar field in three-dimensional black hole spacetime. In the present paper, we provide an explicit expression for the scalar field propagator and the vacuum polarization of $\left\langle\varphi^{2}\right\rangle$ in the Bañados' black hole background in odd dimensions. The calculation of $\left\langle\varphi^{2}\right\rangle$ can be performed by much less effort than the stress tensor. We would regard the calculation of $\left\langle\varphi^{2}\right\rangle$ as a useful preliminary to the calculation of the quantum stress tensor.

In section 2, we briefly review the black hole solutions obtained by Bañados et al to make the present paper self-contained. We obtain the propagator for a conformally coupled massless scalar field in the black hole spacetime in section 3 . The expectation value of $\left\langle\varphi^{2}\right\rangle$ for the scalar field is derived from the propagator. In section 4 we compute $\left\langle\varphi^{2}\right\rangle$ in the five-dimensional black hole spacetime as a concrete example. A summary is given in section 5 .

\section{Black hole solutions in the gravity theory pro- posed by Bañados et al}

We consider $D$-dimensional spacetime, where $D$ is assumed to be odd and written by $D=2 m+1$. The action proposed by Bañados et al, which contains only two coupling constants, is written $b^{2}[14]$ :

$$
I=\kappa_{G} \sum_{p=0}^{m} \frac{a^{-D+2 p}}{D-2 p}\left(\begin{array}{c}
m \\
p
\end{array}\right) I_{p}
$$

with

$$
I_{p}=\int \varepsilon_{a_{1} \ldots a_{D}} R^{a_{1} a_{2}} \wedge \cdots \wedge R^{a_{2 p-1} a_{2 p}} \wedge e^{a_{2 p+1}} \wedge \cdots \wedge e^{a_{D}},
$$

where $\kappa_{G}$ is a dimensionless constant while $a$ is the coupling which has the dimension of length in the natural unit system. We take $\kappa_{G}=1 /(D-2) ! A_{D-2}$ with $A_{D-2}=2 \pi^{(D-1) / 2} \Gamma((D-1) / 2)$. The expression (2) is often referred to as the dimensionally continued Euler form.

When there is no matter field coupled to gravity, the equation of motion is derived from the action as

$$
\varepsilon_{a_{1} \ldots a_{D}} F^{a_{1} a_{2}} \wedge \cdots \wedge F^{a_{D-2} a_{D-1}}=0
$$

where

$$
F^{a b}=R^{a b}+a^{-2} e^{a} \wedge e^{b} .
$$

\footnotetext{
${ }^{2}$ Please be careful about the notation, which differs slightly from Bañados et al.
} 
Bañados et al found the following spherically symmetric solution:

$$
d s^{2}=-g^{2}(r) d t^{2}+g^{-2}(r) d r^{2}+r^{2} d \Omega^{2},
$$

with

$$
g^{2}(r)=1-(1+M)^{1 / m}+\left(\frac{r}{a}\right)^{2}=-\tilde{M}+\left(\frac{r}{a}\right)^{2},
$$

where $M$ is the mass of the black hole. We use the parameter $\tilde{M}$ hereafter for convenience. Note that $\tilde{M}=0$ when $M=0$ and $\tilde{M}$ increases simply with $M$.

For $D=3$, the spacetime is identified as 'anti-de Sitter space with global conical structure'. Therefore we recognize that this spacetime can be made from the anti-de Sitter space with some identification procedure [5]. But for $D \geq 5$, one cannot obtain the metric from the identification process on anti-de Sitter space. In fact, curvature of the spacetime is no longer constant. This feature is akin to the difference between cosmic strings and global monopoles, which induce deficit angles and deficit 'solid' angles respectively.

We can rewrite the metric by using a new coordinate:

$$
r=r_{+} \sec \rho \quad(0 \leq \rho \leq \pi / 2),
$$

where $r_{+}=\sqrt{\tilde{M}} a$.

Then we get

$$
d s^{2}=a^{2}(\sec \rho)^{2}\left(-\kappa^{2} \sin ^{2} \rho d t^{2}+d \rho^{2}+\tilde{M} d \Omega^{2}\right) .
$$

Replacing $t$ with the Euclidean one, we obtain

$$
d s_{E}^{2}=a^{2}(\sec \rho)^{2}\left(\kappa^{2} \sin ^{2} \rho d \tau^{2}+d \rho^{2}+\tilde{M} d \Omega^{2}\right),
$$

where $\tau$ has a period $2 \pi / \kappa$ with $\kappa=\sqrt{\tilde{M}} / a$.

The non-zero components of the Ricci tensor are:

$$
\begin{aligned}
& R_{\tau}^{\tau}=R_{\rho}^{\rho}=-\frac{D-1}{a^{2}}, \\
& R_{n}^{m}=\left[-\frac{D-1}{a^{2}}+\frac{(D-3)(\tilde{M}+1)}{a^{2} \tilde{M}} \cos ^{2} \rho\right] \delta_{n}^{m},
\end{aligned}
$$

where $m$ and $n$ run over the spherical coordinates. One can see the fact that the spacetime expressed by the metric has constant curvature only if $D=3$. The scalar curvature is then

$$
R=\left[-\frac{D(D-1)}{a^{2}}+\frac{(D-2)(D-3)(\tilde{M}+1)}{a^{2} \tilde{M}} \cos ^{2} \rho\right] .
$$

In the next section, we construct the propagator in the Euclidean black hole spacetime in odd dimensions by using the mode sum method. 


\section{Two-point function in the asymptotically anti- de Sitter black hole spacetime}

We introduce a conformally invariant scalar in the Bañados black hole background. The wave equation for a conformal scalar is

$$
\square \varphi-\frac{D-2}{4(D-1)} R \varphi=0,
$$

where the covariant divergence is defined in terms of the background metric (9). The Euclidean propagator $G_{H}$ satisfies:

$$
\left(\square-\frac{D-2}{4(D-1)} R\right) G_{H}\left(x, x^{\prime}\right)=-\frac{1}{\sqrt{\operatorname{det} g_{\mu \nu}}} \delta\left(x, x^{\prime}\right) .
$$

To obtain the propagator, we adopt the mode-sum method. The mode function obeys the wavefunction. If the function takes the form

$$
\varphi_{N n}(x)=u_{N n}(\rho) Y_{N \nu}^{(D-2)}(\Omega) e^{i n \kappa \tau},
$$

where $Y^{(D-2)}(\Omega)$ is the generalized spherical function [2] and $\Omega$ represents the coordinate on a unit $(D 2)$-sphere, the radial function $u_{N n}(\rho)$ obeys

$$
\begin{aligned}
& \frac{\cos ^{D-2} \rho}{\sin \rho} \frac{d}{d \rho} \frac{\sin \rho}{\cos ^{D-2} \rho} \frac{d}{d \rho} u_{N n}(\rho)-\left[\frac{n^{2}}{\sin ^{2} \rho}+\frac{N(N+D-3)}{\tilde{M}}\right] u_{N n}(\rho) \\
& -\frac{D-2}{4(D-1)}\left[-\frac{D(D-1)}{\cos ^{2} \rho}+\frac{(\tilde{M}+1)(D-2)(D-3)}{\tilde{M}}\right] u_{N n}(\rho)=0(.16)
\end{aligned}
$$

The general solution for this differential equation is given by a linear combination of the two independent functions:

$$
u_{N n}(\rho)=(\cos \rho)^{(D-2) / 2}\left[\alpha P_{-1 / 2 \pm i \mu}^{n}(\cos \rho)+\beta Q_{-1 / 2 \pm i \mu}^{n}(\cos \rho)\right],
$$

where $P_{\nu}^{n}(x)$ and $Q_{\nu}^{n}(x)$ are the Legendre functions and

$$
\mu=\sqrt{\frac{1}{\tilde{M}}\left[\left(N+\frac{D-3}{2}\right)^{2}+\frac{(\tilde{M}+1)(D-3)}{4(D-1)}\right]} .
$$

Instead of (17), we will choose a set of real functions $(\cos \rho)^{(D-2) / 2}\left\{P_{-1 / 2+i \mu}^{n}(\cos \rho)\right.$, $\left.P_{-1 / 2+i \mu}^{n}(-\cos \rho)\right\}$ as mode functions.

We can construct the Euclidean propagator from the mode functions. In general, the mode sum takes the following form:

$$
\begin{aligned}
& G_{H}\left(\rho, \tau, \Omega ; \rho^{\prime}, \tau^{\prime}, \Omega^{\prime}\right) \\
& =\frac{\kappa}{2 \pi} \sum_{n=-\infty}^{\infty} e^{i n \kappa\left(\tau-\tau^{\prime}\right)} \sum_{N=0}^{\infty} \sum_{\nu} Y_{N \nu}^{(D-2)}(\Omega) Y_{N \nu}^{(D-2) *}\left(\Omega^{\prime}\right) f\left(\cos \rho_{<}\right) g(\cos \rho(\$))
\end{aligned}
$$


where $\rho_{<}<\rho_{>}$, and, $f$ and $g$ are solutions of equation (16), chosen to reflect boundary conditions.

At $\rho=0$, which corresponds to the horizon, $P_{-1 / 2+i \mu}^{n}(\cos \rho)$ has a finite value and is regular while $P_{-1 / 2+i \mu}^{n}(-\cos \rho)$ and $Q_{-1 / 2+i \mu}^{n}(\cos \rho)$ not. Thus we must take $f \approx(\cos \rho)^{(D-2) / 2} P_{-1 / 2+i \mu}^{n}(\cos \rho)$. At $\rho=-\pi / 2$, which corresponds to spatial infinity, various boundary conditions can be considered, because anti-de Sitter space is not globally hyperbolic [15]. We assume

$$
g(\cos \rho) \approx(\cos \rho)^{(D-2) / 2}\left[P_{-1 / 2+i \mu}^{n}(-\cos \rho)-\alpha P_{-1 / 2+i \mu}^{n}(\cos \rho)\right]
$$

where $\alpha$ is constant. $\alpha=1(-1)$ corresponds to Dirichlet (Neumann) boundary condition at $\rho=\pi / 2$. The condition $\alpha=0$ is called the transparent boundary condition, according to [15].

The normalizations of the mode functions are determined by the Wronskian condition on the two functions. Using the formula

$$
\left|\begin{array}{cc}
P_{-1 / 2+i \mu}^{n}(\cos \rho) & \frac{d}{d \rho} P_{-1 / 2+i \mu}^{n}(\cos \rho) \\
P_{-1 / 2+i \mu}^{n}(-\cos \rho) & \frac{d}{d \rho} P_{-1 / 2+i \mu}^{n}(-\cos \rho)
\end{array}\right|=-\frac{2}{\pi} \cosh \mu \pi \frac{(-1)^{n}}{\sin \rho},
$$

we get the expression for the propagator:

$$
\begin{aligned}
G_{H} & \left(\rho, \tau, \Omega ; \rho^{\prime}, \tau^{\prime}, \Omega^{\prime}\right)=\frac{\kappa}{2 \pi} \sum_{n=-\infty}^{\infty} e^{i n \kappa\left(\tau-\tau^{\prime}\right)} \sum_{N=0}^{\infty} \sum_{\nu} Y_{N \nu}^{(D-2)}(\Omega) Y_{N \nu}^{(D-2) *}\left(\Omega^{\prime}\right) \\
& \times\left(\cos \rho \cos \rho^{\prime}\right)^{(D-2) / 2} \frac{1}{\kappa r_{+}^{D-2}} \frac{\pi}{2} \frac{1}{\cosh \mu \pi} P_{-1 / 2+i \mu}^{n}\left(\cos \rho_{<}\right) \\
& \times\left[P_{-1 / 2+i \mu}^{-n}\left(-\cos \rho_{>}\right)-\alpha P_{-1 / 2+i \mu}^{-n}\left(\cos \rho_{>}\right)\right] .
\end{aligned}
$$

Applying the addition theorem [10] to this then we get

$$
\begin{gathered}
G_{H}\left(\rho, \tau, \Omega ; \rho^{\prime}, \tau^{\prime}, \Omega^{\prime}\right)=\frac{\left(\cos \rho \cos \rho^{\prime}\right)^{(D-2) / 2}}{2 \pi r_{+}^{D-2}} \sum_{N=0}^{\infty} \sum_{\nu} Y_{N \nu}^{(D-2)}(\Omega) Y_{N \nu}^{(D-2) *}\left(\Omega^{\prime}\right) \\
\times \frac{\pi}{2} \frac{1}{\cosh \mu \pi}\left[P_{-1 / 2+i \mu}\left(-\cos \rho \cos \rho^{\prime}-\sin \rho \sin \rho^{\prime} \cos \kappa\left(\tau-\tau^{\prime}\right)\right)\right. \\
\left.-\alpha P_{-1 / 2+i \mu}\left(\cos \rho \cos \rho^{\prime}-\sin \rho \sin \rho^{\prime} \cos \kappa\left(\tau-\tau^{\prime}\right)\right)\right] .(23)
\end{gathered}
$$

Using the integral representation for the Legendre functions [10], we obtain

$$
\begin{gathered}
G_{H}\left(\rho, \tau, \Omega ; \rho^{\prime}, \tau^{\prime}, \Omega^{\prime}\right)=\frac{\left(\cos \rho \cos \rho^{\prime}\right)^{(D-2) / 2}}{2 \pi r_{+}^{D-2}} \sum_{N=0}^{\infty} \sum_{\nu} Y_{N \nu}^{(D-2)}(\Omega) Y_{N \nu}^{(D-2) *}\left(\Omega^{\prime}\right) \\
\times\left[\int_{0}^{\infty} \frac{\cos \mu \phi d \phi}{\sqrt{2\left(\cosh \phi-\cos \rho \cos \rho^{\prime}-\sin \rho \sin \rho^{\prime} \cos \kappa\left(\tau-\tau^{\prime}\right)\right)}}\right. \\
\left.\quad-\alpha \int_{0}^{\infty} \frac{\cos \mu \phi d \phi}{\sqrt{2\left(\cosh \phi+\cos \rho \cos \rho^{\prime}-\sin \rho \sin \rho^{\prime} \cos \kappa\left(\tau-\tau^{\prime}\right)\right)}}\right],(24)
\end{gathered}
$$


where $\mu$ is given by (18).

For $D=3$, this representation is further simplified to (with noting $\tilde{M}=M$ in this case)

$$
\begin{aligned}
& G_{H}\left(\rho, \tau, \theta ; \rho^{\prime}, \tau^{\prime}, \theta^{\prime}\right) \\
& =\sum_{k=-\infty}^{\infty} \frac{(\cos \rho)^{1 / 2}\left(\cos \rho^{\prime}\right)^{1 / 2}}{4 \sqrt{2} \pi a \sqrt{\cosh \sqrt{M}\left(\theta-\theta^{\prime}+2 \pi k\right)-\cos \rho \cos \rho^{\prime}-\sin \rho \sin \rho^{\prime} \cos \kappa\left(\tau-\tau^{\prime}\right)}} \\
& \left.-\alpha \sum_{k=-\infty}^{\infty} \frac{(\cos \rho)^{1 / 2}\left(\cos \rho^{\prime}\right)^{1 / 2}}{\left.4 \sqrt{2} \pi a \sqrt{\cosh \sqrt{M}\left(\theta-\theta^{\prime}+2 \pi k\right)+\cos \rho \cos \rho^{\prime}-\sin \rho \sin \rho^{\prime} \cos \kappa\left(\tau-\tau^{\prime}\right.}\right)}\right)
\end{aligned}
$$

This coincides with the propagator constructed from that in the threedimensional anti-de Sitter space with identification process.

In the next section, we compute the vacuum value for $\left\langle\varphi^{2}\right\rangle$ by using the Euclidean propagator (23) or (24).

\section{Calculation of $\left\langle\varphi^{2}\right\rangle$}

It is easy to calculate $\left\langle\varphi^{2}\right\rangle$ from the Euclidean propagator. The vacuum value $\left\langle\varphi^{2}\right\rangle$ is defined as $[2]$

$$
\left\langle\varphi^{2}\right\rangle(x)=\lim _{x^{\prime} \rightarrow x}\left(G_{H}\left(x, x^{\prime}\right)-G_{H}^{d i v}\left(x, x^{\prime}\right)\right),
$$

where $G_{H}^{d i v}$ denotes the divergent part in the Euclidean propagator. In the spherical black hole background, $\left\langle\varphi^{2}\right\rangle$ is given as a function of $\rho$.

We assume the two points $x$ and $x^{\prime}$ have common values of the coordinates $\tau=\tau^{\prime}$ and $\Omega=\Omega^{\prime}$ : In this case $G_{H}$ becomes a function of $\rho$ and $\rho^{\prime}$.

According to [2], the sum of the spherical functions for $\Omega=\Omega^{\prime}$ can be written as

$$
\sum_{\nu} Y_{N \nu}^{(D-2)}(\Omega) Y_{N \nu}^{(D-2) *}(\Omega)=\frac{(N+m-1) \Gamma(N+2 m-2) \Gamma(m-1)}{2 \pi^{m} \Gamma(N+1) \Gamma(2 m-2)},
$$

where $m=(D-1) / 2$. Using this formula, we find

$$
\begin{gathered}
G_{H}\left(\rho, \rho^{\prime}\right)=\frac{\left(\cos \rho \cos \rho^{\prime}\right)^{m-1 / 2}}{4 \sqrt{2} \pi^{m+1} r_{+}^{2 m-1}} \frac{\Gamma(m-1)}{\Gamma(2 m-2)} \sum_{N=0}^{\infty} \frac{(N+m-1) \Gamma(N+2 m-2)}{\Gamma(N+1)} \\
\quad \times\left[\int_{0}^{\infty} \frac{\cos \mu \phi d \phi}{\sqrt{\cosh \phi-\cos \left(\rho-\rho^{\prime}\right)}}-\alpha \int_{0}^{\infty} \frac{\cos \mu \phi d \phi}{\sqrt{\cosh \phi+\cos \left(\rho-\rho^{\prime}\right)}}\right], \quad \text { (28) }
\end{gathered}
$$

where $\mu$ is given by (3.6)

The denominator of the integrand can be expanded by using the Legendre function:

$$
\frac{1}{\sqrt{2(\cosh \phi-\cos \beta)}}=\sum_{q=0}^{\infty} P_{q}(\cos \beta) e^{-(q+1 / 2) \phi} .
$$


Using this expansion, we carry out the integration over $\phi$ and get:

$$
\begin{aligned}
& G_{H}\left(\rho, \rho^{\prime}\right)=\frac{\left(\cos \rho \cos \rho^{\prime}\right)^{m-1 / 2}}{4 \pi^{m+1} r_{+}^{2 m-1}} \frac{\Gamma(m-1)}{\Gamma(2 m-2)} \sum_{N=0}^{\infty} \frac{(N+m-1) \Gamma(N+2 m-2)}{\Gamma(N+1)} \\
& \quad \times\left[\sum_{q=0}^{\infty} \int_{0}^{\infty}\left(P_{q}\left(\cos \left(\rho-\rho^{\prime}\right)\right)-\alpha P_{q}\left(-\cos \left(\rho+\rho^{\prime}\right)\right)\right) e^{-(q+1 / 2) \phi} \cos \mu \phi d \phi\right] \\
& =\frac{\left(\cos \rho \cos \rho^{\prime}\right)^{m-1 / 2}}{4 \pi^{m+1} r_{+}^{2 m-1}} \frac{\Gamma(m-1)}{\Gamma(2 m-2)} \sum_{N=0}^{\infty} \frac{(N+m-1) \Gamma(N+2 m-2)}{\Gamma(N+1)} \\
& \quad \times\left[\sum_{q=0}^{\infty} \frac{q+1 / 2}{(q+1 / 2)^{2}+\mu^{2}}\left(P_{q}\left(\cos \left(\rho-\rho^{\prime}\right)\right)-\alpha P_{q}\left(-\cos \left(\rho+\rho^{\prime}\right)\right)\right)\right]
\end{aligned}
$$

For odd-dimensional spacetime, the summation on $N$ can be carried out by the technique introduced by [11] and [12].

The expression for $G^{d i v}$, on the other hand, can be found by the method of De Witt [13] and Christensen [14] (and developed by many other authors). The analysis of the point-splitting method is rather simple in our case, because the line element in the radial direction is the same as that in Euclidean anti-de Sitter space.

Now we show the calculation of $\left\langle\varphi^{2}\right\rangle$ for the five-dimensional case $(D=5$, $m=2$ ) as a concrete example. In the higher-dimensional cases, the calculation is tedious but straightforward.

First we rewrite equation (30) by adding a parameter integration, in order to handle the summation over $N$. We consider the following expression:

$$
\begin{gathered}
G_{H}\left(\rho, \rho^{\prime}\right)=\frac{\left(\cos \rho \cos \rho^{\prime}\right)^{3 / 2}}{4 \pi^{3} r_{+}^{3}} \sum_{q=0}^{\infty}\left(q+\frac{1}{2}\right)\left(P_{q}\left(\cos \left(\rho-\rho^{\prime}\right)\right)-\alpha P_{q}\left(-\cos \left(\rho+\rho^{\prime}\right)\right)\right) \\
\times\left[\sum_{N=0}^{\infty}(N+1)^{2} \int_{0}^{\infty} \exp \left\{-\left[\left(q+\frac{1}{2}\right)^{2}+\frac{(N+1)^{2}}{\tilde{M}}+\frac{\tilde{M}+1}{8 \tilde{M}}\right] t\right\} d t\right] . \quad(31)
\end{gathered}
$$

We apply Poisson's summation formula to the sum over $N$. Then we get

$$
\begin{aligned}
& G_{H}\left(\rho, \rho^{\prime}\right)=\frac{\left(\cos \rho \cos \rho^{\prime}\right)^{3 / 2}}{8 \pi^{3} a^{3}} \sqrt{\pi} \sum_{q=0}^{\infty}\left(q+\frac{1}{2}\right)\left(P_{q}\left(\cos \left(\rho-\rho^{\prime}\right)\right)-\alpha P_{q}\left(-\cos \left(\rho+\rho^{\prime}\right)\right)\right) \\
& \times \sum_{L=0}^{\infty} \int_{0}^{\infty} \frac{d t}{t^{3 / 2}}\left(\frac{1}{2}-\frac{\pi^{2} \tilde{M}}{t} L^{2}\right) \exp \left[-\left(q+\frac{1}{2}\right)^{2} t-\frac{\tilde{M}+1}{8 \tilde{M}} t-\frac{\pi^{2} \tilde{M}}{t} L^{2}\right] .
\end{aligned}
$$

In this expression (4.7), we find that the divergent contributions when $\rho \rightarrow \rho^{\prime}$ are contained in the $L=0$ term in the sum.

Taking care of this fact, we integrate the expression over $t$ and obtain:

$$
G_{H}\left(\rho, \rho^{\prime}\right)=\frac{\left(\cos \rho \cos \rho^{\prime}\right)^{3 / 2}}{8 \pi^{2} a^{3}}\left\{\frac{1}{\left[2\left(1-\cos \left(\rho-\rho^{\prime}\right)\right)\right]^{3 / 2}}-\alpha \frac{1}{\left[2\left(1+\cos \left(\rho+\rho^{\prime}\right)\right)\right]^{3 / 2}}\right.
$$




$$
\begin{aligned}
& \left.-\frac{\tilde{M}+1}{16 \tilde{M}}\left[\frac{1}{\sqrt{2\left(1-\cos \left(\rho-\rho^{\prime}\right)\right)}}-\alpha \frac{1}{\sqrt{2\left(1+\cos \left(\rho+\rho^{\prime}\right)\right)}}\right]\right\} \\
& +\frac{\left(\cos \rho \cos \rho^{\prime}\right)^{3 / 2}}{8 \pi^{2} a^{3}} \frac{1}{\sqrt{4 \pi}} \sum_{q=0}^{\infty}\left(P_{q}\left(\cos \left(\rho-\rho^{\prime}\right)\right)-\alpha P_{q}\left(-\cos \left(\rho+\rho^{\prime}\right)\right)\right) \\
& \times\left[\sum_{n=2}^{\infty} \frac{(-1)^{n}}{n !} \frac{\Gamma(n-1 / 2)}{\left.(q+1 / 2)^{2 n-2}\left(\frac{\tilde{M}+1}{8 \tilde{M}}\right)^{n}\right]-\frac{\left(\cos \rho \cos \rho^{\prime}\right)^{3 / 2}}{8 \pi^{2} a^{3}}} 2\right. \\
& \times \sum_{q=0}^{\infty}\left(q+\frac{1}{2}\right) \sqrt{\left(q+\frac{1}{2}\right)^{2}+\frac{\tilde{M}+1}{8 \tilde{M}}\left(P_{q}\left(\cos \left(\rho-\rho^{\prime}\right)\right)\right.} \\
& \left.-\alpha P_{q}\left(-\cos \left(\rho+\rho^{\prime}\right)\right)\right) \frac{1}{\exp \left(2 \pi \sqrt{\tilde{M}} \sqrt{\left(q+\frac{1}{2}\right)^{2}+\frac{\tilde{M}+1}{8 \tilde{M}}}\right)-1} .
\end{aligned}
$$

On the other hand, $G^{d i v}$ can be obtained by the point-splitting method $[13,14]$. In the present case, we take

$$
G_{H}^{d i v}=\frac{\Delta^{1 / 2}}{8 \pi^{2}} \frac{1}{(2 \sigma)^{3 / 2}}\left\{1+\frac{a_{1}}{2}(2 \sigma)+\cdots\right\}
$$

where

$$
\begin{aligned}
\Delta^{1 / 2} & =1+\frac{1}{12} R_{\mu \nu} \sigma^{\mu} \sigma^{\nu}-\frac{1}{24} R_{\mu \nu ; \lambda} \sigma^{\mu} \sigma^{\nu} \sigma^{\lambda}+\cdots, \\
a_{1} & =\left(\frac{1}{6}-\frac{3}{16}\right) R,
\end{aligned}
$$

where $\sigma$ is the geodesic distance and $\sigma_{\mu}=\sigma_{, \mu}$. Now, since we consider the radial separation, we take

$$
\sigma=\frac{a^{2}}{2}\left(\cosh ^{-1} \frac{1-\sin \rho \sin \rho^{\prime}}{\cos \rho \cos \rho^{\prime}}\right)^{2} .
$$

Substituting (35)-(37) into (34), we find $G^{d i v}$ in the limit of small separation, $\Delta \rho=\rho-\rho^{\prime}:$

$$
G^{d i v}\left(\rho, \rho^{\prime}\right)=\frac{1}{8 \pi^{2} a^{3}}\left\{\frac{\cos ^{3} \rho}{\Delta \rho^{3}}+\frac{\cos \rho}{\Delta \rho}\left[-\frac{3}{8}+\frac{\tilde{M}-1}{16 \tilde{M}} \cos ^{2} \rho\right]+O(\Delta \rho)\right\} .
$$

This behaviour for small $\Delta \rho$ turns out to be the same as the small $\Delta \rho$ limit of

$$
\frac{\left(\cos \rho \cos \rho^{\prime}\right)^{3 / 2}}{8 \pi^{2} a^{3}}\left\{\frac{1}{\left[2\left(1-\cos \left(\rho-\rho^{\prime}\right)\right)\right]^{3 / 2}}-\frac{\tilde{M}+1}{16 \tilde{M}} \frac{1}{\sqrt{2\left(1-\cos \left(\rho-\rho^{\prime}\right)\right)}}\right\},
$$

which appears in the two-point function in the five-dimensional anti-de Sitter black hole background (33). 
Consequently, we obtain the expression for the vacuum expectation value $\left\langle\varphi^{2}\right\rangle$ around the Bañados' black hole in five dimensions:

$$
\begin{aligned}
& \left\langle\varphi^{2}\right\rangle(\rho)=\frac{(\cos \rho)^{3}}{8 \pi^{2} a^{3}} \alpha\left[-\frac{1}{[2(1+\cos (2 \rho))]^{3 / 2}}+\frac{\tilde{M}+1}{16 \tilde{M}} \frac{1}{\sqrt{2(1+\cos (2 \rho))}}\right] \\
& +\frac{(\cos \rho)^{3}}{8 \pi^{2} a^{3}} \frac{1}{\sqrt{4 \pi}} \sum_{q=0}^{\infty}\left(1-\alpha P_{q}(-\cos (2 \rho))\right) \\
& \times\left[\sum_{n=2}^{\infty} \frac{(-1)^{n}}{n !} \frac{\Gamma(n-1 / 2)}{\left.(q+1 / 2)^{2 n-2}\left(\frac{\tilde{M}+1}{8 \tilde{M}}\right)^{n}\right]}\right. \\
& -\frac{(\cos \rho)^{3}}{8 \pi^{2} a^{3}} 2 \sum_{q=0}^{\infty}\left(q+\frac{1}{2}\right) \sqrt{\left(q+\frac{1}{2}\right)^{2}+\frac{\tilde{M}+1}{8 \tilde{M}}}\left(1-\alpha P_{q}(-\cos (2 \rho))\right) \\
& \times \frac{1}{\exp \left(2 \pi \sqrt{\tilde{M}} \sqrt{\left(q+\frac{1}{2}\right)^{2}+\frac{\tilde{M}+1}{8 \tilde{M}}}\right)-1} .
\end{aligned}
$$

For $\alpha=0$ (the transparent boundary condition), the dependence on $\rho$ becomes very simple. In terms of the original coordinate $r$ in $(5),\left\langle\varphi^{2}\right\rangle$ is pro-

portional to $r^{-3}$. Therefore using this original coordinate, we can consider continuation of the result to the inside of the black hole. The value of $\left\langle\varphi^{2}\right\rangle$ diverges only at the origin $r=0$ (for any $\alpha$ ).

On the other hand, $\left\langle\varphi^{2}\right\rangle$ approaches zero in the limit of $r \rightarrow \infty$ if and only if $\alpha=0$. The value of $\left\langle\varphi^{2}\right\rangle$ at spatial infinity is a constant, $-\alpha /\left(64 \pi^{2} a^{3}\right)$. This is the value for $\left\langle\varphi^{2}\right\rangle$ in the exact five-dimensional anti-de Sitter space. The numerical evaluation of $8 \pi^{2} a^{3}\left\langle\varphi^{2}\right\rangle(\rho)$ is plotted in figure 1 for $\alpha=-1,0$ and 1 when $\tilde{M}=0.1$ and $\tilde{M}=1$.

The dependence of $\left\langle\varphi^{2}\right\rangle$ at the black hole horizon $\left(\rho=0 ; r=r_{+}\right)$on $\tilde{M}$ is shown in figure 2 . The value of $\left\langle\varphi^{2}\right\rangle$ at the horizon approaches a constant, which is approximately $1 /\left(8 \pi^{2} a^{3}\right)(0.0081-0.099 \alpha)$, in the large $\tilde{M}$ limit. We may remark that this depends only on the length scale $a$.

\section{$5 \quad$ Summary and prospects}

We have obtained a representation for the Euclidean propagator for a conformal scalar field in the asymptotically anti-de Sitter black hole spacetime, which has been found by Bañados et al.

Using the exact propagator, we have computed the vacuum expectation value $\left\langle\varphi^{2}\right\rangle$ for a conformally coupled massless scalar field in the five-dimensional case. The value of $\left\langle\varphi^{2}\right\rangle$ is not positive definite in general.

For large values of the black hole mass, the quantum fluctuation at the black hole horizon does not vanish in the five-dimensional case even if $\mathrm{a}=0$, and perhaps in general higher dimensions. This shows a contrast to the three- 


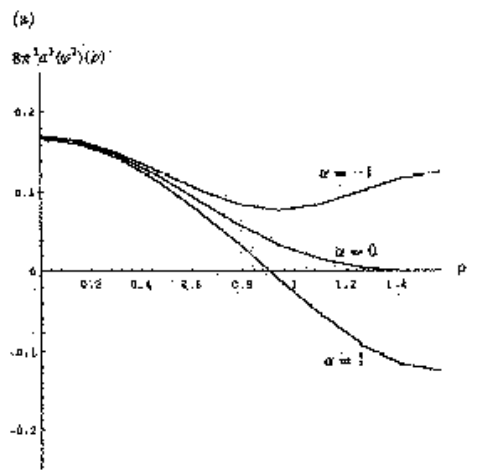

(a)

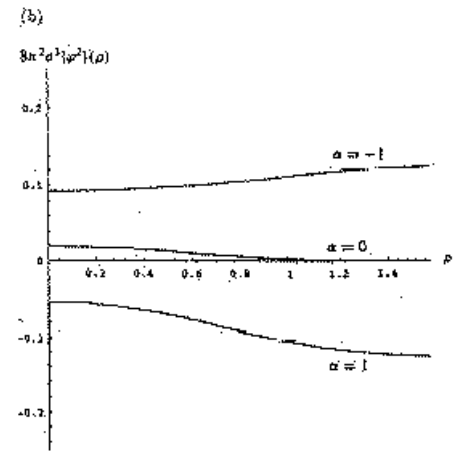

(b)

Figure 1: The magnitude of the vacuum polarization $8 \pi^{2} a^{3}\left\langle\varphi^{2}\right\rangle(\rho)$ as a function of $\rho$ when (a) $\tilde{M}=0.1$ and (b) $\tilde{M}=1$. The curves correspond to $\alpha=-1,0,1$ as indicated.

dimensional case, where the amount of the fluctuation approaches the value of that in the exact anti-de Sitter space.

The amount of quantum fluctuation in the odd-dimensional black hole spacetime cannot be expressed by simple analytic functions of the parameter such as the black hole mass. This is a general feature of field theory in odd dimensions $[11,12]$.

Using the propagator, we should compute the expectation value for the stress tensor of quantum fields near the Bañados black holes as a next step. Then the black hole thermodynamics including quantum fluctuation of the field could be studied. 


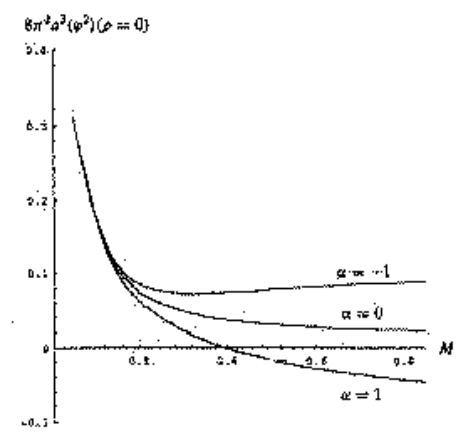

Figure 2: The dependence of the vacuum polarization $8 \pi^{2} a^{3}\left\langle\varphi^{2}\right\rangle$ at the horizon as a function of $\tilde{M}$. The curves correspond to $\alpha=-1,0,1$ as indicated.

\section{References}

[1] P. Candelas, 1980 Phys. Rev. D21 (1980) 2185.

P. Candelas and K. W. Howard, Phys. Rev. D29 (1984) 1618.

K. W. Howard and P. Candelas, Phys. Rev. Lett. 53 (1984) 403.

K. W. Howard, Phys. Rev. D30 (1984) 2532.

V. P. Frolov, Phys. Rev. D26 (1982) 954.

I. D. Novikov and V. P. Frolov, Physics of Black Holes (Dordrecht: Kluwer,1988); Trends in Theoretical Physics vol 2, ed P. J. Ellis and Y. C. Tang (Reading, MA: Addison-Wesley, 1991) pp. 27-75.

P. R. Anderson, Phys. Rev. D39 (1989) 3785; Phys. Rev. D41 (1990) 1152.

[2] [2] V. P. Frolov, F. D. Mazzitelli and J. P. Paz, Phys. Rev. D40 (1989) 948.

[3] For a review and (earlier) references, see: N. D. Birrell and P. C. W. Davies, Quantum Fields in Curved Space (Cambridge: Cambridge University Press, 1982)

[4] M. Bañados, C. Teitelboim and J. Zanelli, Phys. Rev. D49 (1994) 975.

M. Bañados, "Black Holes in Einstein-Lovelock Gravity", CECS Preprint gr-qc/9309011.

[5] M. Bañados, C. Teitelboim and J. Zanelli, Phys. Rev. Lett. 69 (1992) 1849.

M. Bañados, M. Henneaux, C. Teitelboim and J. Zanelli, Phys. Rev. D48 (1993) 1506.

[6] S. F. Ross and R. B. Mann, Phys. Rev. D47 (1993) 3319. 
D. Cangemi, M. Leblanc and R. B. Mann, Phys. Rev. D48 (1993) 3606

A. Achucarro and M. Ortiz, Phys. Rev. 48 (1993) 3600.

G. T. Horowitz and D. L. Welch, Phys. Rev. Lett. 71 (1993) 328.

N. Kaloper, Phys. Rev. D48 (1993) 2598.

C. Farina, J. Gamboa and A. J. Segui-Santonja, Class. Quantum Grav. 10 (1993) L193.

[7] K. Shiraishi and T. Maki, Class. Quantum Grav. 11 (1994) 695; Phys. Rev. D49 (1994) 5286.

[8] A. R. Steif, Phys. Rev. D49 (1994) R585.

[9] G. Lifschytz and M. Ortiz, Phys. Rev. D49 (1994) 1929.

[10] A. Erdelyi et al (ed), Higher Transcendental Functions (New York: McGraw-Hill, 1955)

M. Abramowitz and I. A. Stegun (ed), Handbook of Mathematical Functions (New York: Dover, 1972)

[11] P. Candelas and S. Weinberg, Nucl. Phys. B237 (1984) 397.

[12] M. Yoshimura, Phys. Rev. D30 (1984) 344.

[13] B. S. De Witt, Phys. Rep. 19 (1975) 297.

[14] S. M. Christensen, Phys. Rev. D14 (1976) 2490.

[15] S. J. Avis, C. J. lsham and D. Storey, Phys. Rev. D18 (1978) 3565. 